\title{
Reconstructing Knowledge: Recombinant Strategies for Multicultural Beginning Design Pedagogy
}

\author{
PAVLINA ILIEVA
}

Morgan State University

This paper explores the challenges and benefits of two opposing learning models in Beginning Design education - the experiential versus referential. Reflecting on the development and implementation of the Beginning Design curriculum of the pre-professional Architecture and Environmental Design program at Morgan State University, a minority serving public institution, the author proposes a constructivist learning approach that uses recombinant strategies from the two established pedagogical models.

The reconstructive pedagogy seeks to mediate the gaps between students' diverse academic background, cultural reality of space and goals for the future with traditional architectural pursuits and contemporary disciplinary concerns. In response to the emerging needs of a multicultural design education, key strategies involve: 1) use of students' diverse experiences and overall lack of familiarity with the field as a point of departure for expanding architectural cannon across disciplines, scales and cultures, 2) cultivate habits of the mind and hand that nurture a personal perspective while fostering a culture of rigor and care, 3) distance from high architecture in formal terms in favor of more socially concerned design problems relative to students' motivation for entering the field.

\section{CONTEXT}

As the world settles into a perpetual state of crisis, architecture becomes increasingly viewed as an inevitable victim, a solemn enabler or an urgent remedy to modernity's relentless narrative of disaster, conflict and failure. In response, the architecture field, no longer just the art of making buildings, has expanded into a broad umbrella of problem solving activities with an increased emphasis on the ethical dimension of practice, purpose-driven design intention and the search for solutions, both technical and social ${ }^{1}$. From climate and war disaster aftermath to failed social and financial policies and euphoric technological innovations, the challenges faced by humanity are hardly new but the 'call to action' culture of intimidation and persuasion has thrust the profession into a complex web of interconnected forces. How to prepare designers to address such complexities on the local and global level has been at the forefront of architectural education for the past two decades as evidenced by the social, ecological and technological agenda that has settled in architectural discourse.
Free from the practical implications of 'doing good' and the heroic vision of the 'star architect', architectural education embraced the new millennium with an interdisciplinary design ethos, urge for inclusivity and a bold sense of agency in social, environmental and economic change. The act of implementing these principles, however, has proven challenging as architecture academia struggles to maintain the status quo of the field as fundamental to addressing spatial concerns and the making of buildings while evolving curricula to include the search for solutions to pressing local and global matters. As the diversity of participating constituent on all sides has never been greater, architectural education is called upon to revisit pedagogical strategies that respond to a wider variety of student groups entering the field and also prepare emerging professionals for the ethical challenges of a dynamic, multicultural world.

What are the implications of established Beginning Design pedagogies in the context of the efforts to expand, diversify and evolve the field of architecture as it aims for relevance and contribution to contemporary culture and society? How do we reconstruct one's knowledge of the world in order to equip them with the tools and agency needed to respond to the volatile future ahead?

In a 1993 article "Architecture's Resistance to Diversity: A Matter of Theory as Much as Practice," Linda Groat discusses architecture's symptomatic oscillation between two opposing disciplinary perspectives of theory versus practice, which has contributed to alienating the field and undermining its relevance and influence in society. Her comparative analysis concludes a dead-end for these dueling perspectives and makes a case for adopting a 'culturalist perspective' that embraces principles of diversity and multiculturalism "to achieve a truly respected and valued role in our society." The traditional models of the architect-as-artist and architect-astechnician provide only a limited role in enabling groups with underrepresented gender, ethnic and socioeconomic background to contribute to a more impactful profession ${ }^{2}$. Thus a significant obstacle to effectively transforming architectural education remains the field's ongoing struggle to attract and retain greater diversity of students who are motivated, through their individual circumstance, to seek a meaningful career that can influence positive change in their communities and the world around them. 


\section{THE BEGINNING DESIGN PENDULUM}

When traced back to Walter Gropius and the Bauhaus tradition, Beginning (Basic) Design offers training in modern visual language and space-making techniques. Providing an 'optical key' and operational parameters, he argued, would allow students to approach their design creatively as "limitation obviously makes the creative mind inventive." His ultimate goal was to develop a "common language of visual communication" in order to allow art and architecture to enter everyday modern life and translate further across related disciplines. The method of achieving that relies not on having students absorb and apply disciplinary conventions but rather on immersive exploration, observation and rigorous reaction to formal and spatial phenomena ${ }^{3}$. This marks the beginning of a more egalitarian approach to architectural design where the creative process is neither facilitated by creative genius nor passed on by the master architect but rather discovered, inhabited and realized through rigorous creative pursuits in response to objective, tangible and measurable parameters. It also suggests the study of architectural history and technology later in the curriculum as a matter of support and a source of reference for the creative pursuit.

This pedagogical philosophy favors learning from practical experience rather than from history and responds to the concern of the "innocent" beginning students' individuality being stifled by the conventions of the Beaux-Arts tradition. An opposing philosophy, championed by Joseph Hudnut, Dean of Harvard GSD at the time he and Gropius battled over the introduction of the Basic Design coursework in architectural education, maintained that contemporary architecture is part of the continuum of time. Therefore studying history would allow students to understand the meaning of architecture and learn to think contextually 4 . This approach suggests a different type of learning process - one that is based on assimilating a priori values and knowledge of the field as a matter of positioning the individual's effort in the additive framework of architecture in time.

\section{Experiential Learning}

Contemporary Beginning Design curricula have adopted some version of the experiential introductory approach to architecture rooted in nonrepresentational visual communication and making. The results are often characterized as a relative success due to the challenges faced in subsequent years when transitioning from abstract, procedural and spatial exercises to buildings that involve contextual analysis and technical conventions. This is often the case due to the varied types of competences, specializations and teaching methods of faculty in whose hands falls the ultimate responsibility of delivering continuity and transfer of skills and knowledge throughout the curriculum. One of the main goals of experiential Beginning Design pedagogy is for students to learn about their own interests through creative exploration and discover individual ways of thinking and working within architectural design. It is not uncommon, however, for faculty to find students' Beginning Design preparation inadequate or insufficient for their specific academic agenda, technical or theoretical, so the 'correct' one is promptly introduced.

The leap from abstraction and exploration to the design of buildings rooted in sites and socioeconomic realities remains challenging for students. They either begin to view their Beginning Design studies as unrelated and divorced from the making of buildings and seek more easily accessible paths to generate architectural solutions instead or are not able to reconcile the limitations of available tools and techniques in order to pursue a concept to its full potential. Caught somewhere on the scale of architect as artist or technician, students struggle not only with assuming either occupational identity but also coming to terms with what those mean for them professionally upon degree completion. Their desire to study and address some of the broader issues embedded in the curriculum take the back seat to tackling the fundamental dichotomy of architecture norms. Without meaningful integration between experiential Beginning Design and the remaining architectural curriculum, which targets the main goals that bring students to architecture, the field will continue to struggle with effectively diversifying and transforming the profession.

It is hard to imagine that such thing as a perfectly coordinated curriculum exists. Not only because of potential conflict for academic freedom that a top-down teaching agenda would incur but also because of the inherent value that architecture puts on presenting varied, if not opposing, views for the benefit of advancing a critical dialog about the field. Yet architecture schools rely heavily on presenting a united front on what their contribution to the field and a student's future as an architect would be. Thus, the need to openly embrace contrasting voices and pedagogies can become a fertile ground for a skillful reinterpretation of their delivery and overall impact.

\section{Referential Learning}

The opposing referential approach that favors the study of history, architectural precedent and technical representation as vehicles for establishing a point of departure involves mimetic learning - modeling on examples. Even long after the bruises from post-modern stylistic references have healed, Beginning Design pedagogy and architectural curriculum as a whole are hesitant to recall history for anything more than demonstrating traditional architectural principals or the ill-conceived 'precedent analysis' at the beginning of most studio design projects. While students find general comfort in the ability to draw on examples, their work is under constant pressure for potential lack of originality and preoccupation with derivative styles 5 .

Through this explanation-based approach - safe from abstraction, except for extracting the building's organizational 'parti', 
students are able to develop building-looking designs with relative ease based on a given example. Many students who take architecture technology courses in high school or community college arrive to their first day of architecture class having drafted a small construction set of drawings or modeled a small building of generic design using BIM software and are eager to keep going. Their expectations have already been framed around the technical implications of architecture where design begins with drawing a building.

As history and representation do not get directly involved in design process, Beginning Design pedagogy that substitutes creative exploration with historic precedent and favors technical knowledge over design thinking, develops a particularly narrow initial understanding of architecture. Strong technical skills are favored by mainstream architecture practice and they often get front-loaded in the curriculum with the intention to give students the main tools for a successful career. However, the ability to produce architectural looking content gives a false sense of security to students who are yet to discover that design is a synthetic and not mimetic process and technical knowledge is integral and not alternative to design.

Both experiential and referential approaches to Beginning Design have proven track record of generating or transmitting architectural skills and knowledge to architecture students. The two also present the perpetual disparity between the creative and technical approach in architecture described earlier as well as the gap between academia and practice. It is important to state that these are mostly intra-disciplinary concerns that have more to do with the politics of influence and protecting the status quo of one's personal architectural education rather than a direct response to an evolving field that seeks to address the challenges of the world at large.

As such, every "new" curriculum is bound to carry the genetic material of the ones that shaped its creators. However, through the strategic recombination of curricular fragments from contrasting educational models, standard educational practices can be augmented and leveraged to benefit the learning outcomes for diverse student groups.

\section{CURRICULAR COORDINATION AND CONTINUITY}

Since 2010, the Beginning Design Curriculum at Morgan State University, Bachelor of Science in Architecture and Environmental Design Program has evolved through consistent observation and evaluation of how students engage with varied pedagogical strategies. Prior to and during the initial development process, design exercises on the entire spectrum of the experiential/referential Beginning Design pedagogical pendulum have been introduced at one time or another.

Determining the effectiveness of the exercises was based on annual evaluation and ongoing discussion by course and program faculty based on two main criteria: 1) continuity of specific knowledge and skills in remaining program curriculum and 2) opportunity for students to relate personal interests or experiences during design exploration. More specific content criteria track and evaluate student performance with respect to course and program learning outcomes through portfolio review of high-and low-pass work at the end of each semester.

The 2010-12 period was focused on transitioning from a broadly defined program curriculum with individual faculty introducing varied course objectives in multi-section beginning courses and throughout the remaining course curriculum. During this time, one section of 15 students would engage in redrawing plans and elevations from original drawings with one instructor while another would follow a step-by-step isometric projection drawing of dodecahedron. There was great variety of making exercises among course sections as well from creating an intuitive structure from manila folder that support an empty water bottle in space to building a dome out of paper following detailed instructions. A third section would make nothing at all because the instructor did not find it essential for the introductory design communication courses. Many of the exercises did not pass the continuity or personal exploration criteria test and the rest evolved into parts of the pedagogy we use today. Most importantly, all program faculty embraced the transition to a coordinated approach of multisection instruction of core courses in the first through third years of the curriculum as they all struggled with the varied levels of competence and understanding of students in subsequent studios.

From a continuity standpoint, first year students who engaged mostly in experiential learning in the Beginning Design coursework and did not spend time with architectural drawing conventions had a hard time articulating and representing their design ideas in the second year Foundation Studios. Their design process was among the most original but the work often departed too far from the essential goals and required competencies of the project. Other students who embraced and excelled in the technical aspects of architectural drawing would consider design complete with the execution of a wellcrafted drawing and panic when challenged to continue their exploration and 'change' their design as part of an iterative design process. Both types of responses left students unsatisfied with their performance and proved catching up on various design skills nearly impossible with the rising complexity and demands of upper level studios.

\section{THE SOCIAL DIMENSION}

The 2010-11 student cohort that followed the uncoordinated Beginning Design curriculum has the highest percentage of students passing the first-year coursework (66\%) and the lowest graduation rates ( $22 \%$ for first time freshmen) compared to the inverse trend in more recent years (average 50\% and $40 \%$ respectively). In the context of non-competitive admission to our publically funded University, also an $\mathrm{HBCU}$, and 
below national average graduation rates - 35\% for 6-year graduation period of first time freshmen in 2016, more than $60 \%$ of the incoming students in our program are eligible for Financial Aid and receive Pell Grants ${ }^{6}$. Most first time students drop out from college within their first year due to personal and financial difficulties as well as inadequate preparation for college. These factors are generally unrelated to the students being enrolled in the BS in Architecture and Environmental Design program and are a function of the general demographic and socioeconomic profile of Morgan State University students. In contrast, the only other public BS in Architecture program in our state, which admits freshmen with above 75 percentile SAT scores and significant college level course credit, reported $100 \%$ first year retention of the Fall 2014 cohort and $82 \%$ graduation rate for the Fall 2009 cohort ${ }^{7}$.

While the differences between the 'non-competitive' and 'highly selective' institutions are fairly obvious, there are far more nuanced and consequential conclusions to be drawn, subject to separate comparative analysis. However, it is important to note that despite the low retention and graduation rates at Morgan State University, the number of undergraduate degrees that both programs award annually is nearly the same. Therefore, it is safe to say that considering the Morgan students' average SAT scores and general reliance on Pell Grants to finance their education (tied to in-state tuition), a "highly selective" approach to giving access to architectural education would not allows us to graduate the second largest cohort nationally of African Americans with Bachelor's of Science in Architecture and Environmental Design degree as many of our students would be stopped at the gate of most architecture programs based on entry qualifications alone.

With upper level studio retention and transfer student graduation rates ranging in $80-100 \%$ in recent years, our program has produced competitive, motivated and diverse graduates as evidenced by their wide-ranging career choices and top-ranked employment and graduate admission accomplishments. The issue of selectivity comes up during and after, not before, the first year of design education, which provides students the opportunity to make decisions about their commitment based on personal knowledge and experience with the program. Meanwhile, it is our Beginning Design curriculum that has the unique opportunity and takes on the significant challenge of introducing a multicultural cohort of students with varied personal motivation and preparation to the architecture and built environment field.

\section{RECOMBINANT PEDAGOGIES}

The coordinated Fall 2012 Beginning Design curriculum coincided with the School of Architecture and Planning moving into a brand new, state-of-the-art, LEED Platinum certified facility after years of occupying substandard spaces with limited access to technology. The program used the renewed sense of optimism and greater resources to build community capacity among students, faculty and the local profession. The goal of the revised first year pedagogy was never to increase the number of students who continue beyond but rather to equip them with varied tools, experiences and knowledge that can sustain their continued growth in the program.

\section{Questioning the Beginning}

Students are not clean slates. This is a common misconception and an essential premise to the idea of reconstructing knowledge. Apart from prior related education experience and relative exposure to the field, they come with multitude of preconceptions about themselves, the world as they know it and architecture as they have imagined it. Added to that are often unreliable pre-existing learning and working habits that determine the students' general ability to function in the first weeks of class.

Upon entry surveys and discussions, it becomes clear that students of diverse ethnic, racial and socioeconomic background are rarely drawn to the field by, and even hardly aware of, iconic architecture, famous architects and disciplinary record. Most incoming students at Morgan express desire for cultural impact through their creative pursuits, making a difference in their communities or influencing broader economic, social and ecological disparities. The lack of familiarity with the established buildings, architects and histories of the field provides an opportunity to offer a more inclusive cannon of practitioners, thinkers and advocates that reflect a multicultural outlook on contemporary practice.

\section{Theory as Practice}

In ARCH 101 Concepts and Theories of the Built Environment, students explore two thematic aspects of architecture and the built environment - 'Place Matters' and 'Place Makers'. The latter introduces specific disciplines and individuals, women
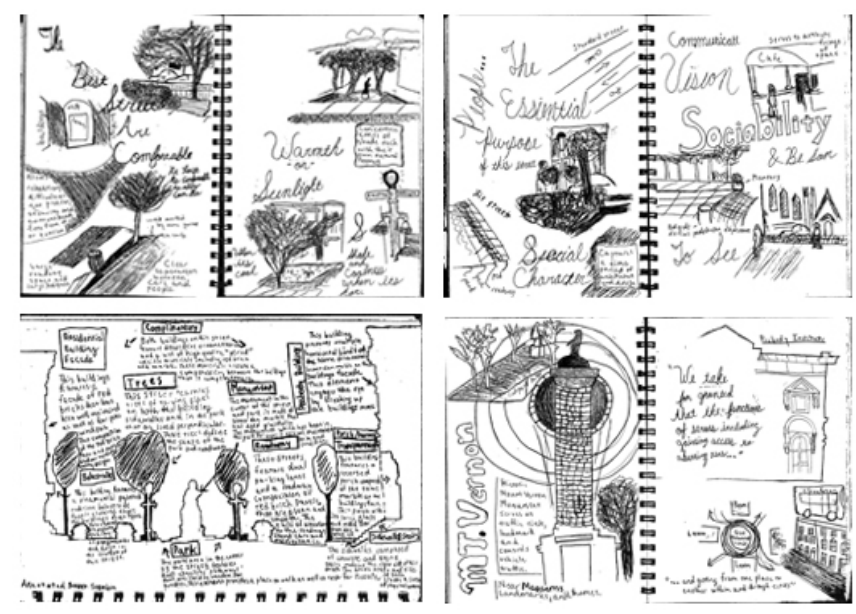

Figure 1: Illustrative journal entry analysis of place making in Mount Vernon Historic Neighborhood of Baltimore. Concepts and Theory of the Built Environment, Fall 2014, Instructor: Gabriel Kroiz. 

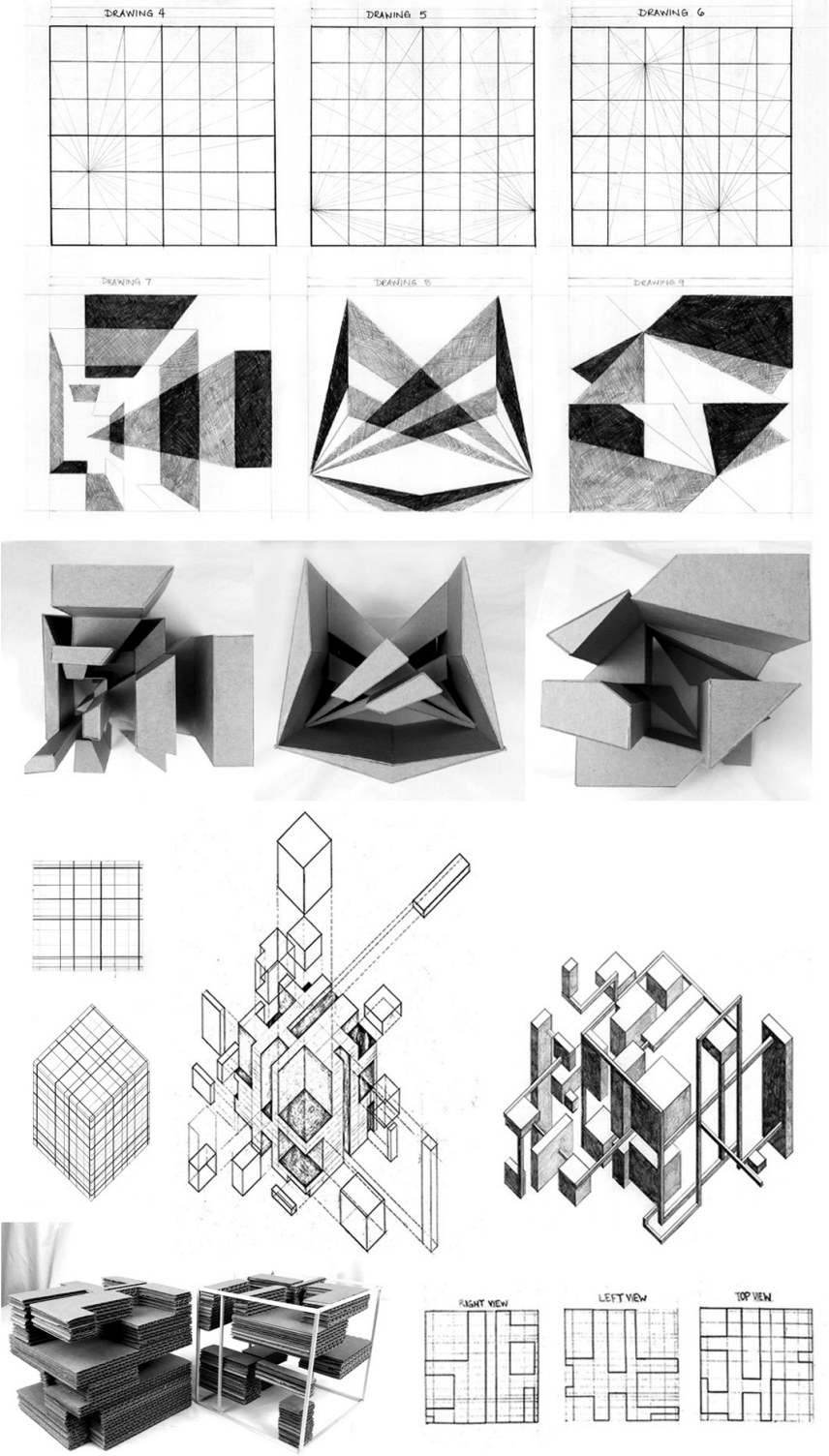

Figure 2: Graphic and built explorations of grid, layers, solid \& void, addition \& subtraction and intuitive structure that translate to architectural space, drawings and models while introducing basic graphic conventions, fundamental design processes and architectural conventions. Communication Skills I \& II, Fall \& Spring 2013-15, Instructor: Pavlina Ilieva.

and men of color, who are involved in the production of places that we inhabit. Students are expected to gain insight both on the character of the individual and their work as well as the formative events and experiences during their advancement in their field. Basic research methods and illustrative hand-drawn poster allow students with limited knowledge and visual communication skills to begin to situate people and places that they identify with in the disciplinary continuum of training, practice and collective experience.

Other projects introduce primary research skills with the rigorous observation and documentation of two familiar places and the subsequent analysis of the qualities that make them
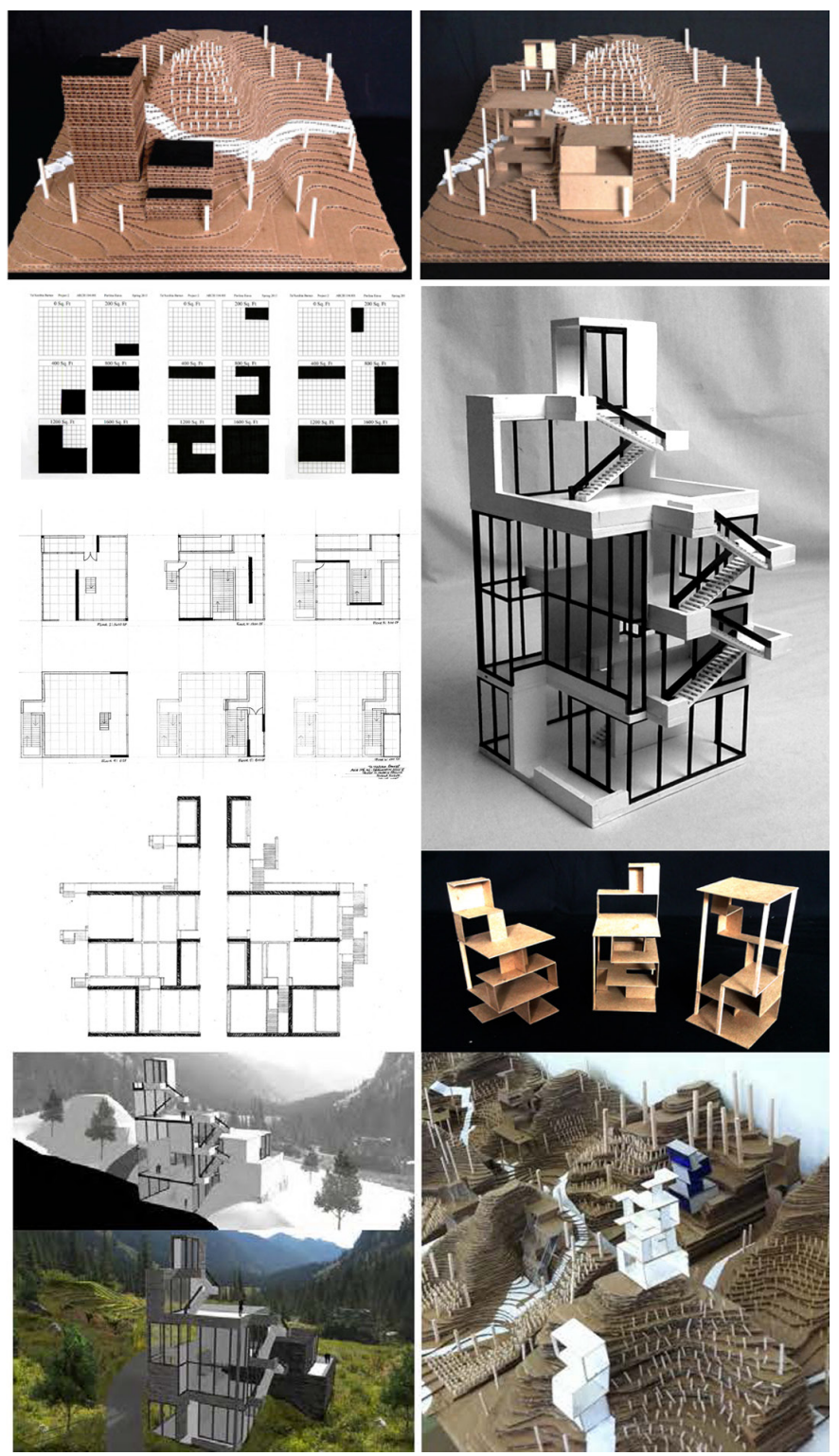

'a place worth caring' and 'a place not worth caring" about. Assumptions about what makes a successful place are tested next in sketch journal analysis of a historic neighborhood (Figure 1) and a short video exploration of the architectural and ephemeral elements that contribute to the 'sense of place' of a public space in the city.

Starting with the students' present understanding of their environment, these experiential exercises reconstruct new knowledge and build intellectual confidence through personal observation and critical inquiry. In-class lectures and demonstrations, readings and videos from established and contemporaneous sources serve as referential devices common to the history/theory course type.

Practice as Theory

A pair of Design Communication courses in each semester of the first year provides the primary visual communication 

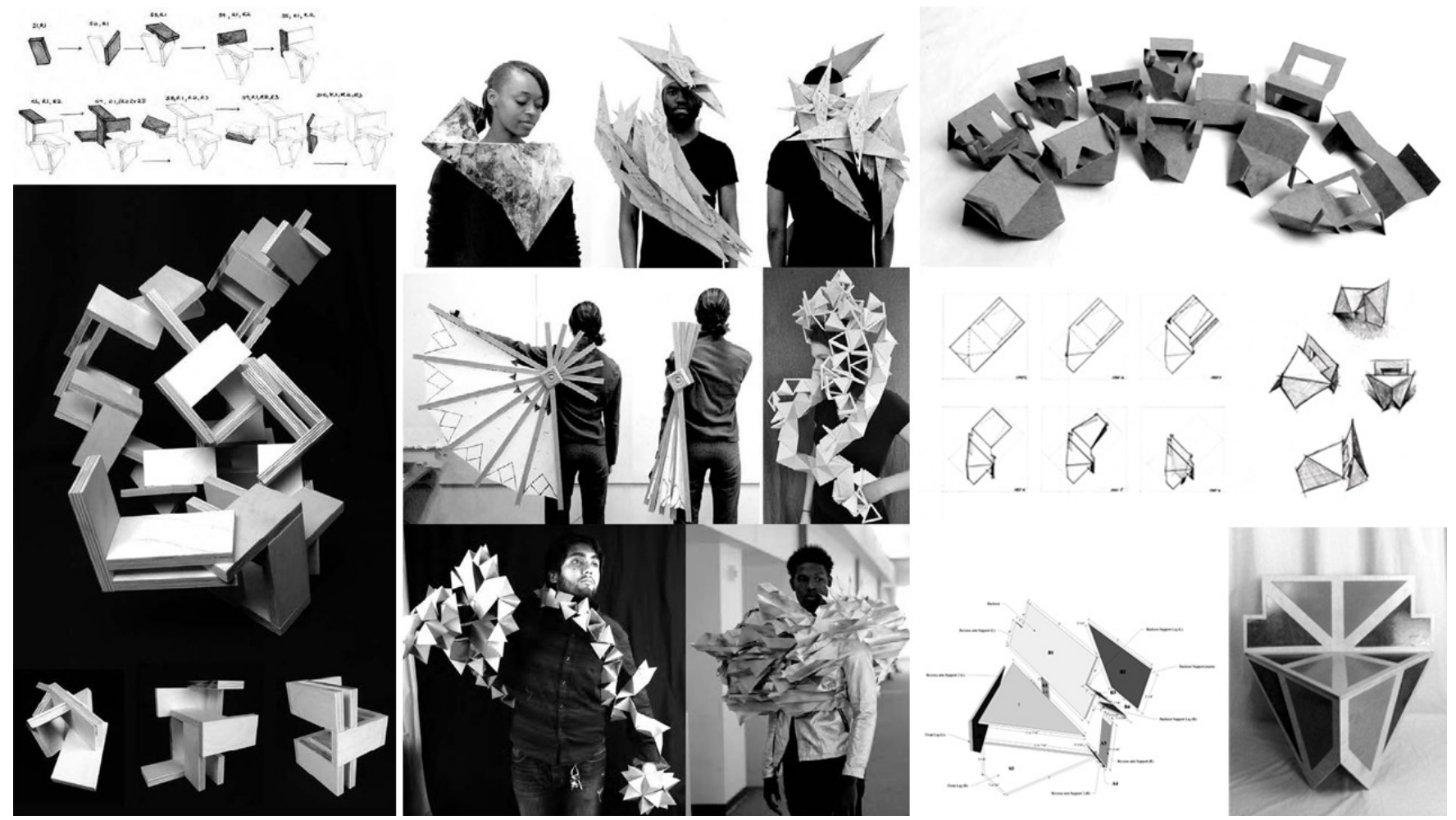

Figure 3: Generative procedural studies and their step-by-step aggregation transition to a starting point of the Wearable Architecture project and the iterative Chair project 'folded' from a single sheet of plywood. Design Communication Skills I \& II, 2013-17, Instructor: Pavlina Ilieva.

and making skill training of the Beginning Design curriculum. Through a series of hands-on tectonic and spatial exercises students are introduced to basic hand drafting, projection drawing types, model building, woodworking, lasercutting and digital documentation techniques (Figure2). Each project starts with an 'a priori' concept (Grid, Mass, Module, etc.) that students explore using spatial operations (Layering, Subtraction, Assemblage) and observe resulting spatial conditions (Emergence, Solid/Void, Aggregation). The experiential portion of the course is augmented by in-class lectures and discussions that position each of the concepts and processes in context of practical and theoretical architectural pursuits and in reference to other design disciplines, visual arts, nature and technology. As project topics address familiar notions of great complexity, students are challenged to revisit their understanding based on observations they have made within the proposed framework and to draw connections among various scales and contexts in an effort to establish a synthetic understanding of something ubiquitous and obvious.

The oscillation between the ordinary and unfamiliar helps make a strong case for design as a means of translation between ideas and reality, using analogy to native topics in order to awaken critical insight. These concepts require no extensive scaffolding and rely on associative conversations about their application, limitations and possibilities during the course of the project. Exercises prescribe a few short initials steps that provide the main framework for exploration and development of students' spatial understanding, vocabulary and dexterity.

All drawing and making techniques are reinforced in the second semester Design Communications course where the notions of layered grids become floors of a tower, subtractive volumes become site and massing models and procedural aggregations lead to iterative investigations (Figure 3). As demonstrated in multiple examples from contemporary and traditional sources, the courses seek to establish that architectural design is a practice. As such, it relies on building familiarity and confidence through repetition, such as in sports and music, until patterns of thinking and actions become habitual, 'second nature'. Meanwhile these early design courses provide a field of ideas for exploration and positioning of one's interest and capacity, rather than immediate and sustained enculturation in architectural cannon. History and theory are introduced as context for exploration much how drawing and making utilize a set of basic parameters to control outcomes.

\section{Communication}

The Concepts and Theories course concludes the first semester as each student identifies, documents, analyzes and recommends improvements for a site in an undervalued city neighborhood by seeking to leverage an opportunity or neutralize a threat. In Design Communications, the same cohort is asked to design, construct and a piece architecture that transforms the proportions of the body and enables or restricts its 
movement. The final projects aim at positioning our students not only as working professionals but also stakeholders and agents of change in their community by embracing various levels of civic and professional engagement while celebrating the newfound skills and attitudes of a designer through public performance and display.

\section{CONCLUSION}

The recombinant Beginning Design pedagogy considers each student as a designer fully immersed in the environment they can observe and act upon regardless of their general unfamiliarity with the field. Students are encouraged to engage their performative capacities immediately by introducing them to matters and tools that help reconstruct their engagement with prior knowledge and understanding in order to develop ways of thinking and working within architectural design.

As the field envelops sweeping agenda that urges the design of new policy, realignment of disciplinary connections between various fields and reevaluation of cultural habits and norms, Beginning Design pedagogies that embrace recombinant strategies would allow architecture academia to attract and retain a more diverse student body and train more impactful professionals, motivated by and ready to address their own spatial understanding as it relates to the ethical imperatives of our time.

\section{ENDNOTES}

1 Timothy Hyde, "Architecture, Modernity, Crisis," in Journal of Architectural Education 69:1 (2015): 2-3.

2 Linda Groat, "Architecture's Resistance to Diversity: A Matter of Theory as Much as Practice," in Journal of Architectural Education 47:1 (1993): 3-10.

3 Walter Gropius, "Re:Education of an Architect," Gropius Papers, Archives of American Art, Smithsonian Institution (1939).

4 Jill Pearlman, "Inventing American Modernism: Joseph Hudnut, Walter Gropius, and the Bauhaus Legacy at Harvard," University of Virginia Press (2007).

5 Amanda Reeser Lawrence, "Radical Acts of Influence: Thoughts on Anxiety, History and the Culture of the Copy," Journal of Architectural Education 69:1 (2015): 13-15.

6 Morgan State University Institutional Data, 2017.

7 University of Maryland, Office of Institution Research, Planning \& Assessment, last modified February, 2016, https://www.irpa.umd.edu/CampusCounts/ CollegeProfiles/ARCH.pdf. 